International Journal of Pure and Applied Mathematics

Volume 92 No. 4 2014, 471-480

ISSN: 1311-8080 (printed version); ISSN: 1314-3395 (on-line version)

url: http://www.ijpam.eu

doi: http://dx.doi.org/10.12732/ijpam.v92i4.3

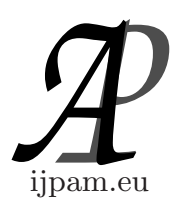

\title{
A NOTE ON UPPER AND LOWER WEAKLY QUASI CONTINUOUS FUZZY MULTIFUNCTIONS
}

\author{
Anjana Bhattacharyya \\ Department of Mathematics \\ Victoria Institution (College) \\ 78 B, A.P.C. Road, Kolkata, 700009, INDIA
}

\begin{abstract}
We have already introduced upper and lower (weakly) quasi continuous fuzzy multifunctions in [3] ([3]). In [8], Malakar introduced fuzzy $\theta$-continuous multifunctions. Again Mukherjee and Malakar have introduced fuzzy almost continuous multifunctions [9]. In this paper we have established a mutual relationships among these fuzzy multifunctions.
\end{abstract}

AMS Subject Classification: 54A40, 54C99

Key Words: fuzzy almost regular space, fuzzy almost preopen multifunction, $\theta$-cluster point of a fuzzy set

This paper is a continuation of [3]. A fuzzy multifunction is a function which carries a point of an ordinary topological space $X$ to a fuzzy set in a fuzzy topological space $Y$ according to Papageorgiou [13] in 1985. After introducing fuzzy multifunction by Papageorgiou, a good many researchers have been inspired to study it and a number of fuzzy multifunctions have been introduced and studied. Papageorgiou defined fuzzy upper and lower inverse of a fuzzy multifunctions. But the definition of lower inverse was not suitable for further study so that Mukherjee and Malakar [9] redefined it suitably in 1991 and this

Received: August 3, 2013

(c) 2014 Academic Publications, Ltd. url: www.acadpubl.eu 
new definition of lower inverse has been accepted to many of the researchers. In this paper we take the definition of upper inverse by Papageorgiou and the definition of lower inverse by Mukherjee and Malakar.

Throughout this paper, by $(X, \tau)$ or simply by $X$ we shall mean an ordinary topological space, while $\left(Y, \tau_{Y}\right)$ or simply $Y$ stands for a fuzzy topological space (fts, for short) in the sense of Chang [4]. The support of a fuzzy set $A$ in $Y$ will be denoted as supp $A$ [17] and is defined by supp $A=\{y \in Y: A(y) \neq 0\}$. A fuzzy point [15] with the singleton support $y \in Y$ and the value $\alpha(0<\alpha \leq 1)$ at $y$ will be denoted by $y_{\alpha} .0_{Y}$ and $1_{Y}$ are the constant fuzzy sets taking respectively the constant values 0 and 1 on $Y$. The complement of a fuzzy set $A$ in $Y$ will be denoted by $1-A[17]$. For two fuzzy sets $A$ and $B$ in $Y$, we write $A \leq B$ iff $A(y) \leq B(y)$, for each $y \in Y$, while we write $A q B$ to mean $A$ is quasi-coincident (q-coincident, for short) with $B$ [15] if there exists $y \in Y$ such that $A(y)+B(y)>1$; the negation of $A q B$ is written as $A \bar{q} B . \operatorname{cl} A$ and int $A$ of a set $A$ in $X$ (respectively, a fuzzy set $A$ [17] in $Y$ ) respectively stand for the closure and interior of $A$ in $X$ (respectively, in $Y$ ). A fuzzy set $A$ in $Y$ is called fuzzy regular open [1] if intcl $A=A$. The complement of a fuzzy regular open set is called fuzzy regular closed [1]. A fuzzy set $B$ is called a quasi neighbourhood ( $q$-nbd, for short) of a fuzzy set $A$ in an fts $Y$ if there is a fuzzy open set $U$ in $Y$ such that $A q U \leq B$. If, in addition, $B$ is fuzzy open (regular open) then $B$ is called a fuzzy open (regular open) $q$-nbd of $A$. In particular, a fuzzy (open) set $B$ in $Y$ is a fuzzy open (resp. regular open) $q$-nbd of a fuzzy point $y_{\alpha}$ in $Y$ if $y_{\alpha} q U \leq B$, for some fuzzy open set $U$ in $Y$. For a subset $A$ of a topological space $X$, the $\theta$-interior of $A$, denoted by $\theta-i n t A$ [16], is defined to be the set of those points $x$ of $X$ such that there exists an open nbd $U_{x}$ of $x$ with $c l U_{x} \subseteq A$. A set $A$ (or a fuzzy set $A$ ) in a topological space $X$ (in an fts $Y$ ) is said to be semiopen [7] (fuzzy semiopen [1]) if there exists an open set (respectively a fuzzy open set) $U$ in $X$ (in $Y$ ) such that $U \subseteq A \subseteq \operatorname{cl} U$ (resp. $U \leq A \leq c l U)$, or equivalently, if $A \subseteq \operatorname{clint} A$ (resp. $A \leq \operatorname{clint} A)$. By $S O(X)$ (resp. $F S O(Y)$ ) we mean the set of all semiopen (resp. fuzzy semiopen) sets in $X$ (in $Y$ ). The complement of a semiopen set (resp. fuzzy semiopen set) in $X$ (resp. in $Y$ ) is called a semiclosed (fuzzy semiclosed) set. We mean semiclosure (resp. fuzzy semiclosure) of a set $A$ in $X$ (resp. of a fuzzy set $A$ in $Y$ ), to be written as $\operatorname{scl} A$, which is the union of all points (resp. fuzzy points) $x$ in $X$ (resp. $y_{\alpha}$ in $Y$ ) such that for every semiopen set (resp. fuzzy semiopen set) $U$ in $X$ (in $Y$ ) with $x \in U$ (resp. $y_{\alpha} q U$ ), it follows that $U \cap A \neq \phi[5]$ (resp. $U q A$ [6]). A set $A$ in $X$ (resp. a fuzzy set $A$ in $Y$ ) is semiclosed (fuzzy semiclosed) iff $A=\operatorname{scl} A$. The union of all semiopen (resp. fuzzy semiopen) sets in $X$ (resp. in $Y$ ) contained in a set (resp. fuzzy set) $A$ is called the semi-interior (resp. 
fuzzy semi-interior) of $A$, denoted by $\operatorname{sint} A$. It follows from definition that a set (resp. a fuzzy set) $A$ is semiopen (resp. fuzzy semiopen) iff $A=\operatorname{sint} A$. A set $B$ in $X$ is called a seminbd of a set $A$ in $X$ if there exists a semiopen set $U$ in $X$ such that $A \subseteq U \subseteq B$.

Let us now recall the following definition from [13].

Definition 1. Let $(X, \tau)$ and $\left(Y, \tau_{Y}\right)$ be respectively an ordinary topological space and an fts. We say that $F: X \rightarrow Y$ is a fuzzy multifunction if corresponding to each $x \in X, F(x)$ is a unique fuzzy set in $Y$.

Henceforth by $F: X \rightarrow Y$ we shall mean a fuzzy multifunction in the above sense.

Definition 2. (see [13] and [9]) For a fuzzy multifunction $F: X \rightarrow Y$, the upper inverse $F^{+}$and lower inverse $F^{-}$are defined as follows:

For any fuzzy set $A$ in $Y, F^{+}(A)=\{x \in X: F(x) \leq A\}$ and $F^{-}(A)=$ $\{x \in X: F(x) q A\}$.

There is a following relationship between the upper and the lower inverses of a fuzzy multifunction.

Theorem 3. (see [9]) For a fuzzy multifunction $F: X \rightarrow Y$, we have $F^{-}(1-A)=X-F^{+}(A)$, for any fuzzy set $A$ in $Y$.

We now recall the following two definitions for ready references.

Definition 4. (see [3]) A fuzzy multifunction $F: X \rightarrow Y$ is said to be:

(a) fuzzy upper weakly quasi continuous (f.u.w.q.c., for short) (resp. fuzzy upper quasi continuous, f.u.q.c., for short) at a point $x \in X$ if for each open set $U$ in $X$ containing $x$ and each fuzzy open set $V$ in $Y$ containing $F(x)$, there exists a non-empty open set $G$ in $X$ such that $G \subseteq U$ and $F(G) \leq c l V$ (resp. $F(G) \leq V)$,

(b) fuzzy lower weakly quasi continuous (f.l.w.q.c., for short) (resp. fuzzy lower quasi continuous, f.l.q.c., for short) at a point $x \in X$ if for each open set $U$ in $X$ containing $x$ and each fuzzy open set $V$ in $Y$ with $F(x) q V$, there exists a non-empty open set $G$ in $X$ such that $G \subseteq U$ and $F(g) q c l V$ (resp. $F(g) q V$ ), for each $g \in G$,

(c) f.u.w.q.c. (f.l.w.q.c.) on $X$ if $F$ has the corresponding property at each point $x$ of $X$.

Definition 5. (see [2]) A fuzzy multifunction $F: X \rightarrow Y$ is said to be:

(a) fuzzy upper almost quasi continuous (f.u.a.q.c., for short) at a point $x \in X$, if for each open set $U$ in $X$ containing $x$ and each fuzzy open set $V$ in 
$Y$ containing $F(x)$, there exists a non-empty open set $G$ in $X$ such that $G \subseteq X$ and $F(G) \leq s c l V$,

(b) fuzzy lower almost quasi continuous (f.l.a.q.c., for short) at a point $x \in X$, if for each open set $U$ in $X$ containing $X$ and each fuzzy open set $V$ in $Y$ with $F(x) q V$, there exists a non-empty open set $G$ in $X$ such that $G \subseteq U$ and $F(g) q s c l V$, for all $g \in G$,

(c)f.u.a.q.c. (f.l.a.q.c.) on $X$ if $F$ has the corresponding property at each point $x$ of $X$.

We now recall the following two theorems from [2].

Theorem 6. A fuzzy multifunction $F: X \rightarrow Y$ is f.u.a.q.c. iff $F^{+}(V) \in$ $S O(X)$, for every fuzzy regular open set $V$ in $Y$.

Theorem 7. A fuzzy multifunction $F: X \rightarrow Y$ is f.l.a.q.c. iff $F^{-}(V) \in$ $S O(X)$, for every fuzzy regular open set $V$ in $Y$.

We recall the following two theorems from [2] for ready references.

Theorem 8. A fuzzy multifunction $F: X \rightarrow Y$ is f.u.a.q.c. at a point $x$ of $X$ iff for any fuzzy open set $V$ in $Y$ containing $F(x)$, there exists $U \in S O(X)$ with $x \in U$ such that $F(U) \leq s c l V$.

Theorem 9. A fuzzy multifunction $F: X \rightarrow Y$ is f.l.a.q.c. at a point $x$ of $X$ iff $F^{-}(V) \in S O(X)$, for every fuzzy regular open set $V$ in $Y$.

Definition 10. (see [10]) An fts $X$ is said to be fuzzy almost regular if for each fuzzy point $x_{\alpha}$ in $X$ and for each fuzzy regular open $q$-nbd $U$ of $x_{\alpha}$, there exists a fuzzy regular open $q$-nbd $V$ of $x_{\alpha}$ such that $c l V \leq U$.

Theorem 11. If $F: X \rightarrow Y$ is f.l.w.q.c. and $Y$ is fuzzy almost regular then $F$ is f.l.a.q.c.

Proof. Let $V$ be a fuzzy regular open set in $Y$ and $x \in F^{-}(V)$. Then $F(x) q V$. Since $Y$ is fuzzy almost regular, there exists a fuzzy regular open set $W$ in $Y$ such that $F(x) q W \leq c l W \leq V$. Since $F$ is f.l.w.q.c., there exists a semiopen set $U_{x}$ in $X$ containing $x$ such that $F(u) q c l W$, for all $u \in U_{x} \Rightarrow$ $F(u) q V$, for all $u \in U_{x}$. Therefore, we have $x \in U_{x} \subseteq F^{-}(V)$. This implies that $F^{-}(V) \in S O(X)$ and hence by Theorem $9, F$ is f.l.a.q.c.

Definition 12. A fuzzy multifunction $F: X \rightarrow Y$ is said to be fuzzy almost propen if $F(U) \leq \operatorname{int}(\operatorname{cl}(F(U)))$ for every $U \in S O(X)$.

Theorem 13. If $F: X \rightarrow Y$ is fuzzy upper weakly quasi continuous and fuzzy almost preopen, then it is fuzzy upper almost quasi continuous. 
Proof. For any $x \in X$ and any fuzzy open set $V$ of $Y$ containing $F(x)$, there exists $U \in S O(X)$ with $x \in U$ such that $F(U) \leq c l V$ (as $F$ is f.u.w.q.c.). Since $F$ is fuzzy almost preopen, $F(U) \leq \operatorname{int}(c l(F(U))) \leq \operatorname{int}(c l V)=\operatorname{scl} V$ (as $V$ is fuzzy open in $Y$ ) [2]. Hence by Theorem $8, F$ is f.u.a.q.c.

Theorem 14. Let $F: X \rightarrow Y$ be a fuzzy multifunction such that $F(x)$ is fuzzy open in $Y$ for each $x \in X$. Then the following are equivalent:

(a) $F$ is fuzzy lower quasi continuous.

(b) $F$ is fuzzy lower almost quasi continuous.

(c) $F$ is fuzzy lower weakly quasi continuous.

Proof. (a) $\Rightarrow$ (b) $\Rightarrow$ (c) are obvious. We only show that (c) $\Rightarrow$ (a).

(c) $\Rightarrow$ (a) : Let $x \in X$ and $V$ be a fuzzy open set in $Y$ such that $F(x) q V$. Since $F$ is f.l.w.q.c., there exists $U \in S O(X)$ containing $x$ such that $F(u) q c l V$ for every $u \in U$. Since $F(u)$ is fuzzy open in $Y$ by the given condition, $F(u) q V$ for every $u \in U$ and hence (a) follows.

Remark 15. It is clear that f.u.a.q.c. (f.u.q.c.) $\Rightarrow$ f.u.w.q.c. and f.l.a.q.c. (f.l.q.c.) $\Rightarrow$ f.l.w.q.c., though the converses are not always true, as the following examples clarify.

Example 16. Let $X=\{a, b, c\}, Y=[0,1], \tau=\{\phi, X\}, \tau_{Y}=\left\{0_{Y}, 1_{Y}, A, B\right\}$ where $A(y)=0.35, B(y)=.04$ for all $y \in Y$. Let $F:(X, \tau) \rightarrow\left(Y, \tau_{Y}\right)$ be a fuzzy multifunction defined by $F(a)=A, F(b)=B, F(c)=C$ where $C(y)=0.6$, for all $y \in Y$. Now $1_{Y}$ and $B$ are only non-null fuzzy regular open sets in $\left(Y, \tau_{Y}\right)$ and $F^{+}(B=\{x \in X: F(x) \leq B\}=\{a, b\} \notin S O(X)$ and so $F$ is not f.u.a.q.c. by Theorem 6 . Now $F^{+}(\operatorname{cl} B)=F^{+}(1 \backslash B)=\{x \in X: F(x) \leq 1 \backslash B\}=X$. Therefore $F^{+}(B) \subseteq \operatorname{cl}\left(\operatorname{int}\left(F^{+}(\operatorname{cl} B)\right)\right)$. Now $F^{+}(A)=\{a\}$. Again $F^{+}(\operatorname{cl} A)=$ $F^{+}(1 \backslash B)=X$ and so $F^{+}(A) \subseteq \operatorname{cl}\left(\operatorname{int}\left(F^{+}(\operatorname{cl} A)\right)\right.$. Hence $F$ is f.l.w.q.c.

Since $F$ is not f.u.a.q.c. it is not f.u.q.c.

Example 17. Let $X=\{a, b, c\}, Y=[0,1], \tau=\{\phi, X,\{a\}\}, \tau_{Y}=$ $\left\{0_{Y}, 1_{Y}, B\right\}$ where $B(y)=0.4$, for all $y \in Y$. Then $1_{Y}$ and $B$ are the only fuzzy regular open sets in $\left(Y, \tau_{Y}\right)$. let $F:(X, \tau) \rightarrow\left(Y, \tau_{Y}\right)$ be a fuzzy multifunction defined by $F(a)=A, F(b)=B, F(c)=C$ where $A(y)=0.45$, $C(y)=0.61$, for all $y \in Y$. Now $F^{-}(B)=\{x \in X: F(x) q B\}=\{c\} \notin S O(X)$. Hence $F$ is not f.l.a.q.c. by Theorem 7. But $F^{-}(c l B)=F^{-}(1 \backslash B)=\{x \in$ $X: F(x) q(1 \backslash B)\}=\{a, c\}$. Therefore, $\operatorname{cl}\left(\operatorname{int}\left(F^{-}(\operatorname{cl} B)\right)\right)=X$ and hence $F$ is f.l.w.q.c.

Since $F$ is not f.l.a.q.c. it is not f.l.q.c. 
We now recall the following definition from [6] for ready reference.

Definition 18. A fuzzy multifunction $F: X \rightarrow Y$ is called:

(a) fuzzy upper $\theta$-continuous (f.u. $\theta$.c., for short) at some point $x_{0} \in X$ iff for every fuzzy open set $V$ in $Y$ with $x_{0} \in F^{+}(V)$, there exists an open nbd $U$ of $x_{0}$ such that $c l U \subseteq F^{+}(c l V)$,

(b) fuzzy lower $\theta$-continuous (f.l. $\theta$.c., for short) at some point $x_{0} \in X$ iff for every fuzzy open set $V$ in $Y$ with $x_{0} \in F^{-}(V)$, there exists an open nbd $U$ of $x_{0}$ such that $c l U \subseteq F^{-}(c l V)$.

We now recall following two theorems from [6].

Theorem 19. For a fuzzy multifunction $F: X \rightarrow Y$, the following are equivalent:

(a) $F$ is f.l. $\theta$.c. on $X$, for each fuzzy open set $V$ in $Y, F^{-}(V) \subseteq \theta-$ $\operatorname{int}\left(F^{-}(c l V)\right)$,

(c) $\theta-\operatorname{cl}\left(F^{+}(\right.$int $\left.V)\right) \subseteq F^{+}(V)$, for any fuzzy closed set $V$ in $Y$.

Theorem 20. For a fuzzy multifunction $F: X \rightarrow Y$, the following are equivalent:

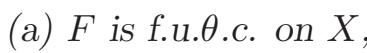

(b) for each fuzzy open set $V$ in $Y, F^{+}(V) \subseteq \theta-\operatorname{int}\left(F^{+}(c l V)\right)$,

(c) $\theta-\operatorname{int}\left(F^{-}(\operatorname{int} V)\right) \subseteq F^{-}(V)$, for any fuzzy closed set $V$ in $Y$.

We now recall following two theorems from [3] for ready references.

Theorem 21. For a fuzzy multifunction

$$
F: X \rightarrow Y, \quad F^{+}(G) \subseteq \operatorname{cl}\left(\operatorname{int}\left(F^{+}(c l G)\right)\right)
$$

for every fuzzy open set $G$ in $Y$.

Theorem 22. For a fuzzy multifunction

$$
F: X \rightarrow Y, \quad F^{-}(G) \subseteq \operatorname{cl}\left(\operatorname{int}\left(F^{-}(c l G)\right)\right)
$$

for every fuzzy open set $G$ of $Y$.

Remark 23. It is clear from Definition 18 and Definition 4 that f.u. $\theta$.c.

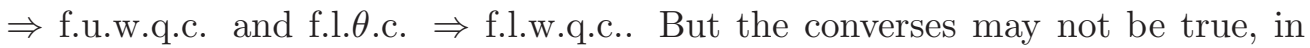
general, as the following examples clarify. 
Example 24. Let $X=\{a, b, c\}, Y=[0,1], \tau=\{\phi, X,\{a\},\{c\},\{a, c\}\}$, $\tau_{Y}=\left\{0_{Y}, 1_{Y}, A, B\right\}$ where $A(y)=0.35, B(y)=0.4$, for all $y \in Y$. Let $F:(X, \tau) \rightarrow\left(Y, \tau_{Y}\right)$ be a fuzzy multifunction defined by $F(a)=A, F(b)=B$, $F(c)=C$ where $C(y)=0.61$, for all $y \in Y$. Now closed sets in $X$ are $\phi$, $X,\{b\},\{b, c\},\{a, b\}$. Now $F^{+}(A)=\{x \in X: F(x) \leq A\}=\{a\}$. Now $c l A=1 \backslash B$. Therefore $F^{+}(c l A)=F^{+}(1 \backslash B)=X \backslash F^{-}(B)$. Now $F^{-}(B)=$ $\{x \in X: F(x) q B\}=\{c\}$ Therefore $F^{+}(c l A)=X \backslash\{c\}=\{a, b\}$. Therefore, $\operatorname{cl}\left(\operatorname{int}\left(F^{+}(\operatorname{cl} A)\right)\right)=\operatorname{cl}(\operatorname{int}(\{a, b\}))=\operatorname{cl}(\{a\})=\{a, b\}$. Therefore $F^{+}(A) \subseteq$ $\operatorname{cl}\left(\operatorname{int}\left(F^{+}(\operatorname{cl} A)\right)\right)$.

$F^{+}(B)=\{x \in X: F(x) \leq B\}=\{a, b\} . \quad F^{+}(c l B)=F^{+}(1 \backslash B)=$ $\{a, b\}$ (by above). Therefore, $\operatorname{cl}\left(\operatorname{int}\left(F^{+}(\operatorname{cl} B)\right)\right)=\{a, b\}$. Therefore, $F^{+}(B) \subseteq$ $c l\left(\operatorname{int}\left(F^{+}(c l B)\right)\right)$. Hence $F$ is f.u.w.q.c. by Theorem 21 .

But $\theta-\operatorname{int}\left(F^{+}(c l B)\right)=\theta-\operatorname{int}(\{a, b\} \not \ngtr b$. Indeed, the only open nbd of $b$ in $X$ is $X$ and so $c l X=X \nsubseteq\{a, b\}$. Therefore, $F^{+}(B) \nsubseteq \theta-\operatorname{int}\left(F^{+}(c l B)\right)$. Hence $F$ is not f.u. $\theta$.c. by Theorem 20 .

Example 25. Let $X=\{a, b, c\}, Y=[0,1], \tau=\left\{\phi, X,\{a\}, \tau_{Y}=\right.$ $\left\{0_{Y}, 1_{Y}, B\right\}$ where $B(y)=0.4$, for all $y \in Y$. Let $F:(X, \tau) \rightarrow\left(Y, \tau_{Y}\right)$ be a fuzzy multifunction defined by $F(a)=A, F(b)=B, F(c)=C$ where $A(y)=0.45$, $C(y)=0.61$, for all $y \in Y$. Now $F^{-}(B)=\{x \in X: F(x) q B\}=\{c\}$ and $\operatorname{cl}\left(\operatorname{int}\left(F^{-}(\operatorname{cl} B)\right)\right)=\operatorname{cl}\left(\operatorname{int}\left(F^{-}(1 \backslash B)\right)\right)=\operatorname{cl}\left(\operatorname{int}\left(X \backslash F^{+}(B)\right)\right)$. Now $F^{+}(B)=$ $\{x \in X: F(x) \leq B\}=\{b\}$. Therefore, $\left.\operatorname{cl}\left(\operatorname{int}\left(F^{-}(\operatorname{cl} B)\right)\right)=\operatorname{cl}(\operatorname{int}(X \backslash\{b\}))\right)=$ $\operatorname{cl}(\operatorname{int}(\{a, c\}))=\operatorname{cl}(\{a\})=X . F^{-}(B) \subseteq \operatorname{cl}\left(\operatorname{int}\left(F^{-}(c l B)\right)\right)$. Hence $F$ is f.l.w.q.c. by Theorem 21 .

But $\theta-\operatorname{int}\left(F^{-}(c l B)\right)=\theta-\operatorname{int}(\{a, c\}) \not \ngtr c$. Indeed, $X$ is the only one nbd of $c$ in $X$ and so $c l X=X \nsubseteq\{a, c\}$. Hence $F^{-}(B) \nsubseteq \theta-\operatorname{int}\left(F^{-}(c l B)\right)$ where $B$ is fuzzy open in $Y$. Hence $F$ is not f.l. .c. by Theorem 19 .

Remark 26. Fuzzy upper (lower) quasi continuity and fuzzy upper (lower) $\theta$-continuity are independent notions, as the following four examples clarify.

Example 27. Let $X=\{a, b, c\}, Y=[0,1], \tau=\{\phi, X,\{a\},\{c\},\{a, c\}\}$, $\tau_{Y}=\left\{0_{Y}, 1_{Y}, A, B\right\}$ where $A(y)=0.35, B(y)=0.4$, for all $y \in Y$. Let $F:(X, \tau) \rightarrow\left(Y, \tau_{Y}\right)$ be a fuzzy multifunction defined by $F(a)=A, F(b)=B$, $F(c)=C$ where $C(y)=0.61$, for all $y \in Y$. Here $F$ is not f.u. $\theta$.c. But $F$ is f.u.q.c. Indeed, $F^{+}(A)=\{a\} \subseteq \operatorname{cl}\left(\operatorname{int}\left(F^{+}(A)\right)\right)=\operatorname{cl}(\operatorname{int}(\{a\}))=\operatorname{cl}(\{a\}=$ $\{a, b\}$. And $F^{+}(B)=\{a, b\}=\operatorname{cl}(\operatorname{int}(\{a, b\}))=\operatorname{cl}(\{a\})=\{a, b\}$.

Example 28. Let $X=\{a, b, c\}, Y=[0,1], \tau=\{\phi, X\}, \tau_{Y}=\left\{0_{Y}, 1_{y}, A, B\right\}$ where $A(y)=0.35, B(y)=0.4$, for all $y \in Y$. Let $F:(X, \tau) \rightarrow\left(Y, \tau_{Y}\right)$ be a fuzzy multifunction defined by $F(a)=A, F(b)=B, F(c)=C$ where $C(y)=0.6$, for all $y \in Y$. Here it is clear that $F$ is f.u. $\theta$.c. But $F$ is not f.u.q.c. 
In fact, $F^{+}(B)=\{a, b\}$ and $\operatorname{cl}\left(\operatorname{int}\left(F^{+}(B)\right)\right)=\operatorname{cl}(\operatorname{int}(\{a, b\}))=\phi$. Therefore, $F^{+}(B) \nsubseteq \operatorname{cl}\left(\operatorname{int}\left(F^{+}(B)\right)\right)$ though $B$ is fuzzy open in $Y$.

Example 29. Consider Example 25. We see that $F$ is f.l.q.c. As $F^{-}(A)=$ $\phi$ and hence $F^{-}(A) \subseteq \operatorname{cl}\left(\operatorname{int}\left(F^{-}(A)\right)\right)$. Also $F^{-}(B)=\{c\}$ and $\operatorname{cl}\left(\operatorname{int}\left(F^{-}(B)\right)\right)=$ $c l(\operatorname{int}(\{c\}))=c l(\{c\})=\{b, c\}$. Therefore, $F^{-}(B) \subseteq \operatorname{cl}\left(\operatorname{int}\left(F^{-}(B)\right)\right)$. But $F$ is not f.l. $\theta$.c. Since $F^{-}(\operatorname{cl} B)=F^{-}(1 \backslash B)=X \backslash F^{+}(B)=X \backslash\{a, b\}=\{c\}$. Therefore, $\theta-\operatorname{int}\left(F^{-}(c l B)\right)=\theta-\operatorname{int}(\{c\}) \ni c$ (Indeed, $\{c\},\{a, c\}, X$ are the only open nbds of $\{c\}$ and $\operatorname{cl}(\{c\})=\{b, c\} \nsubseteq\{c\}, \operatorname{cl}(\{a, c\})=X \nsubseteq\{c\}$ and $c l X=X \nsubseteq\{c\})$. Hence the result.

Example 30. let $X=\{a, b, c\}, Y=[0,1], \tau=\{\phi, X,\{a, c\},\{b\}\}, \tau_{Y}=$ $\left\{0_{Y}, 1_{Y}, B\right\}$ where $B(y)=0.4$, for all $y \in Y$. Let $F:(X, \tau) \rightarrow\left(Y, \tau_{Y}\right)$ be a fuzzy multifunction defined by $F(a)=A, F(b)=B, F(c)=C$ where $A(y)=0.45$, $C(y)=0.61$, for all $y \in Y$. Now $F^{-}(B)=\{x \in X: F(x) q B\}=\{c\}$ and $\operatorname{cl}\left(\operatorname{int}\left(F^{-}(B)\right)\right)=\operatorname{cl}(\operatorname{int}(\{c\}))=\phi$. Therefore, $F^{-}(B) \nsubseteq \operatorname{cl}\left(\operatorname{int}\left(F^{-}(B)\right)\right)$ and hence $F$ is not f.l.q.c. But $F^{-}(c l B)=F^{-}(1 \backslash B)=X \backslash F^{+}(B)=X \backslash\{b\}=\{a, c\}$. and $c \in \theta-\operatorname{int}\left(F^{-}(c l B)\right)$ as $\{a, c\}$ is an open nbd of $c$ such that $c l(\{a, c\})=$ $\{a, c\}=F^{-}(c l B)$. Therefore, $F^{-}(B) \subseteq \theta-\operatorname{int}\left(F^{-}(c l B)\right)$ and hence $F$ is f.l. $\theta$.c.

Remark 31. Fuzzy upper (lower) $\theta$-continuity and fuzzy upper (lower) almost quasi continuity are independent notions.

Example 32. Let $X=\{a, b, c\}, Y=[0,1], \tau=\{\phi, X\}, \tau_{Y}=\left\{0_{Y}, 1_{Y}, A, B\right\}$ where $A(y)=0.35, B(y)=0.4$, for all $y \in Y$. Let $F:(X, \tau) \rightarrow\left(Y, \tau_{Y}\right)$ be a fuzzy multifunction defined by $F(a)=A, F(b)=B, F(c)=C$ where $C(y)=0.61$, for all $y \in Y$. Here $F$ is not f.u.a.q.c. But $F^{+}(A)=\{a\}$ and $\theta-\operatorname{int}\left(F^{+}(c l A)\right)=\theta-i n t\left(F^{+}(1 \backslash B)\right)=\theta-i n t\left(X \backslash F^{-}(B)\right)=\theta-i n t(X \backslash\{c\})=$ $\theta-\operatorname{int}(\{a, b\})=\phi$ and therefore $F^{+}(A) \nsubseteq \theta-\operatorname{int}\left(F^{+}(c l A)\right)$. Hence $F$ is not f.u. $\theta$.c.

Example 33. Consider Example 24. Here $F$ is not f.u. $\theta$.c. Here $B$ is the only non-null fuzzy regular open set in $Y$ other than $1_{Y} \cdot F^{+}(B)=\{a, b\} \in$ $S O(X)$ as $\{a\} \in \tau$ with $\{a\} \subseteq\{a, b\} \subseteq \operatorname{cl}(\{a\})=\{a, b\}$. Hence $F$ is f.u.a.q.c.

Example 34. Consider Example 30. Here $1_{Y}$ and $B$ are the only non-null fuzzy regular open sets in $\left(Y, \tau_{Y}\right)$. Now $F^{-}(B)=\{c\} \notin S O(X)$ and hence $F$ is not f.l.a.q.c. But $F^{-}(c l B)=F^{-}(1 \backslash B)=X \backslash F^{+}(B)=X \backslash\{b\}=\{a, c\}$. Now $\{a, c\}$ is an open nbd of $c$ and $c l(\{a, c\})=\{a, c\}=F^{-}(c l B)$. Therefore, $c \in \theta-\operatorname{int}\left(F^{-}(c l B)\right)$ and so $F^{-}(B) \subseteq \theta-\operatorname{int}\left(F^{-}(c l B)\right)$. Hence $F$ is f.l. $\theta$.c.

Example 35. Let $X=\{a, b, c\}, Y=[0,1], \tau=\{\phi, X,\{a, c\},\{c\}\}, \tau_{Y}=$ $\left\{0_{Y}, 1_{Y}, B\right\}$ where $B(y)=0.4$ for all $y \in Y$. Then $B$ is the only regular open set 
in $Y$ other than $0_{Y}$ and $1_{Y}$. Let $F:(X, \tau) \rightarrow\left(Y, \tau_{Y}\right)$ be a fuzzy multifunction defined by $F(a)=A, F(b)=B, F(c)=C$ where $A(y)=0.45, C(y)=0.61$, for all $y \in Y$. Now $F^{-}(B)=\{x \in X: F(x) q B\}=\{c\} \in S O(X)$ and hence $F$ is f.l.a.q.c. But $F^{-}(c l B)=F^{-}(1 \backslash B)=X \backslash F^{+}(B)=X \backslash\{b\}=\{a, c\}$. Now $\{a, c\}$ and $\{c\}$ are the only proper open nbd of $c$ in $X$ with $\operatorname{cl}(\{c\})=X$ $=c l(\{a, c\})$ so that $X \nsubseteq\{a, c\}$ and hence $c \notin \theta-\operatorname{int}\left(F^{-}(c l B)\right)$. Therefore,

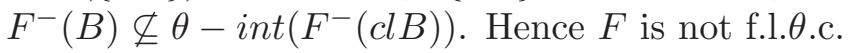

Finally we have got the following diagram:

f.u.q.c. $\Rightarrow$ f.u.a.q.c. $\Rightarrow$ f.u.w.q.c. $\Leftarrow$ f.u. $\theta$.c.

f.l.q.c. $\Rightarrow$ f.l.a.q.c. $\Rightarrow$ f.l.w.q.c. $\Leftarrow$ f.l.t.c.

\section{References}

[1] Azad, K.K., On fuzzy semicontinuity, fuzzy almost continuity and fuzzy weakly continuity, Jour. Math. Anal. Appl., 82 (1981), 14-32.

[2] Bhattacharyya, Anjana, Concerning almost quasi continuous fuzzy multifunctions, Universitatea Din Bacău Studii Si Cercetări Stiintifice Seria: Matematică, 11 (2001), 35-48.

[3] Bhattacharyya, Anjana, Upper and lower weakly quasi continuous fuzzy multifunctions, Analele Universitătii Oradea Fasc. Matematica, TOM XX, Issue No. 2 (2013),5-17.

[4] Chang, C.L., Fuzzy topological spaces, J. Math. Anal. Appl., 24 (1968), 182-190.

[5] Crossley, S.G. and Hildebrand, S.K., Semiclosure, Texas Jour. Scien., 22 (1971), 99-112.

[6] Ghosh, B., Semi continuous and semiclosed mappings and semiconnectedness in fuzzy setting, Fuzzy sets and Systems, 35 (1990), 345-355.

[7] Levine, N., Semiopen sets and semicontinuity in topological spacea, Amer Math. Monthly, 70 (1963), 36-41.

[8] Malakar, S., $\theta$-continuous fuzzy set valued functions on topological spaces, Bull. Cal. Math. Soc., 85 (1993), 541-548. 
[9] Mukherjee, M.N. and Malakar, S., On almost continuous and weakly continuous fuzzy multifunctions, Fuzzy Sets and Systems, 41 (1991), 113-125.

[10] Mukherjee, M.N. and Sinha, S.P., On some near fuzzy continuous functions between fuzzy topological spaces, Fuzzy Sets and Systems, 34 (1990), 245254.

[11] Njåstad, O., On some classes of nearly open sets, Pacific Jour. Math., 15 (1965), 961-970.

[12] Noiri, T., On S-closed subspaces, Atti Accad. Naz. Lincai. Rend. Cl. Sci. Fis. Mat. Natur., 8, c64c (1978), 157-162.

[13] Papageorgiou, N.S., Fuzzy topology and fuzzy multifunctions, Jour. Math. Anal. Appl., 109 (1985), 397-425.

[14] Popa, V., On upper and lower weakly quasi-continuous multifunctions, Rev. Romaine Math. Pures Appl., 27 (1992), 499-508.

[15] Pu, Pao Ming and Liu, Ying Ming, Fuzzy topology I. Neighbourhood structure of a fuzzy point and Moore-Smith convergence, Jour. Math. Anal. Appl., 76 (1980), 571-599.

[16] Velicko, N.V., H-closed topological spaces, Amer. Math. Soc. Trans., 78 (1968), 103-118.

[17] Zadeh, L.A., Fuzzy Sets, Inform. Control, 8 (1965), 338-353. 